\title{
The influence of thermal activation and the intrinsic temperature dependence of the spin torque effect in current-induced domain wall motion
}

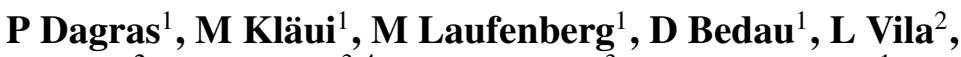 \\ G Faini ${ }^{2}$, C A F Vaz ${ }^{3,4}$, J A C Bland ${ }^{3}$ and U Rüdiger ${ }^{1}$ \\ ${ }^{1}$ Fachbereich Physik, Universität Konstanz, Universitätsstraße 10, 78457 Konstanz, Germany \\ ${ }^{2}$ Laboratoire de Photonique et de Nanostructures - CNRS, Route de Nozay, 91460 \\ Marcoussis, France \\ ${ }^{3}$ Cavendish Laboratory, University of Cambridge, Madingley Road, Cambridge, \\ CB3 0HE, UK \\ E-mail: mathias.klaeui@uni-konstanz.de
}

Received 8 August 2006, in final form 3 November 2006

Published 16 February 2007

Online at stacks.iop.org/JPhysD/40/1247

\begin{abstract}
An experimental study of domain wall motion in $\mathrm{Ni}_{80} \mathrm{Fe}_{20}$ ring structures induced by current pulses as well as conventional magnetic fields is presented. Using constrictions we demonstrate that current-induced domain wall motion can be used to displace walls into parts of the structure where no pulsed currents are flowing. Measurements at variable temperatures between 2 and $300 \mathrm{~K}$ show that the fields necessary for wall motion decrease with increasing temperature, which can be explained by thermal activation. For the current-induced case we find, depending on the geometry and temperature range, that the current densities necessary for displacement can increase or decrease with rising temperature. This indicates that, in addition to thermal excitations, an intrinsic temperature dependence of the efficiency of the spin torque effect is present and leads to an increase in the critical current density with increasing temperature.
\end{abstract}

(Some figures in this article are in colour only in the electronic version)

Domain walls and reversal by domain wall motion have recently become the focus of interest by allowing us to address fundamental physical questions, such as the geometry dependent spin structure $[1,2]$, pinning of domain walls at constrictions [3-6] and the details of the domain wall propagation processes [7,8]. Additionally, devices based on domain walls have been suggested for storage and logic $[9,10]$. Rather than using conventional magnetic fields to move domain walls, recently current-induced domain wall motion [11-22] has received much attention, since it opens up a route for simple device fabrication, where no field-generating strip lines are necessary. Devices based on current-induced domain wall motion have also been put

${ }^{4}$ Present address: Applied Physics, Yale University, New Haven, CT 06520, USA. forward, the most prominent being the racetrack memory [20]. Apart from possible applications, the interplay between spin currents and domain walls in magnetic nanostructures is of fundamental interest, since the basic physical mechanisms involved are not completely understood. The phenomenon of current-induced domain wall motion has been long known theoretically [11, 12] as well as experimentally [13], and recently controlled current-induced motion of single domain walls in magnetic nanostructures has been achieved. Several important aspects such as domain wall velocities [14, 15], critical current densities [16-18,22], thermally assisted motion [19] and the deformation of the domain wall spin structure due to current [15] have been addressed but so far these studies have been mostly limited to vortex walls. While current-induced domain wall motion is experimentally 
well established, the underlying theory of interaction between current and magnetization is still controversial. Different approaches have been suggested, in the ballistic limit [23,24] as well as in the diffusive limit [12,24]. The assumption that the spin of the charge carriers follows the local magnetization leads to the introduction of an adiabatic torque into the Landau-Lifshitz-Gilbert equation of magnetization dynamics [24-26]. Motivated by large discrepancies between experiment and theory, a non-adiabatic term was introduced [27,28]. He et al [29] predicted that the (non)adiabaticity parameter of the spin torque strongly influences the combinations of field and current necessary to move a wall and so a study of domain wall motion as a function of current and field might help to ascertain this parameter if simulations and experimental results are available for the same geometry. Furthermore, a discrepancy between room temperature measurements and $0 \mathrm{~K}$ calculations of the domain wall velocity exists $[14,15,27,28]$. To reveal possible explanations, a temperature-dependent study of the critical current density is a key issue to determine the temperature dependence of the spin torque effect efficiency.

In the field-induced case, domain wall motion is thermally assisted [30], but the effects of thermal activation on currentinduced domain wall propagation have so far only been studied theoretically in the limit of a rigid wall approach and for small excitations at current densities just below the threshold [31]. Spinwaves have been neglected in the $0 \mathrm{~K}$ theory of spin torque, but theoretically the current was shown to alter the spinwave spectrum [32,33]. Thus, to understand the interplay between spin torque and thermal effects and to obtain information on the non-adiabaticity of the torque, current- and field-induced domain wall propagation experiments at different temperatures are needed.

In this paper, we present our recent results of currentand field-induced domain wall motion for transverse and vortex walls at cryostat temperatures between 2 and $300 \mathrm{~K}$ for different geometries and domain wall types. We study the movement of domain walls in nanostructures with constrictions (which generate an attractive potential well) and in structures with no artificial geometrical variations to study the effects of thermal activation and the intrinsic dependence of the domain wall motion due to spin torque on temperature.

Permalloy $\left(\mathrm{Ni}_{80} \mathrm{Fe}_{20}\right)$ rings with thicknesses between 12 and $34 \mathrm{~nm}$, outer diameters between 1 and $2 \mu \mathrm{m}$ and widths of 100 and $200 \mathrm{~nm}$ with and without constrictions have been fabricated. Using a two-step lithography process as described in [34] non-magnetic Au contacts have been lithographically defined.

A scanning electron microscopy (SEM) image of a $12 \mathrm{~nm}$ thick and $200 \mathrm{~nm}$ wide ring with an outer diameter of $2 \mu \mathrm{m}$ and 2 constrictions is shown in figure 1(a). Compared with wires, the ring geometry has the advantage that a domain wall can easily be generated and positioned by applying a homogeneous magnetic field [35]. Domain walls in such structures are head-to-head or tail-to-tail $180^{\circ}$ domain walls with either a vortex or a transverse spin structure $[1,2,36]$. For this particular geometry, the domain wall type was determined to be a transverse wall using magnetoresistance measurements as detailed in [37], which is also expected from the phase diagram [2].
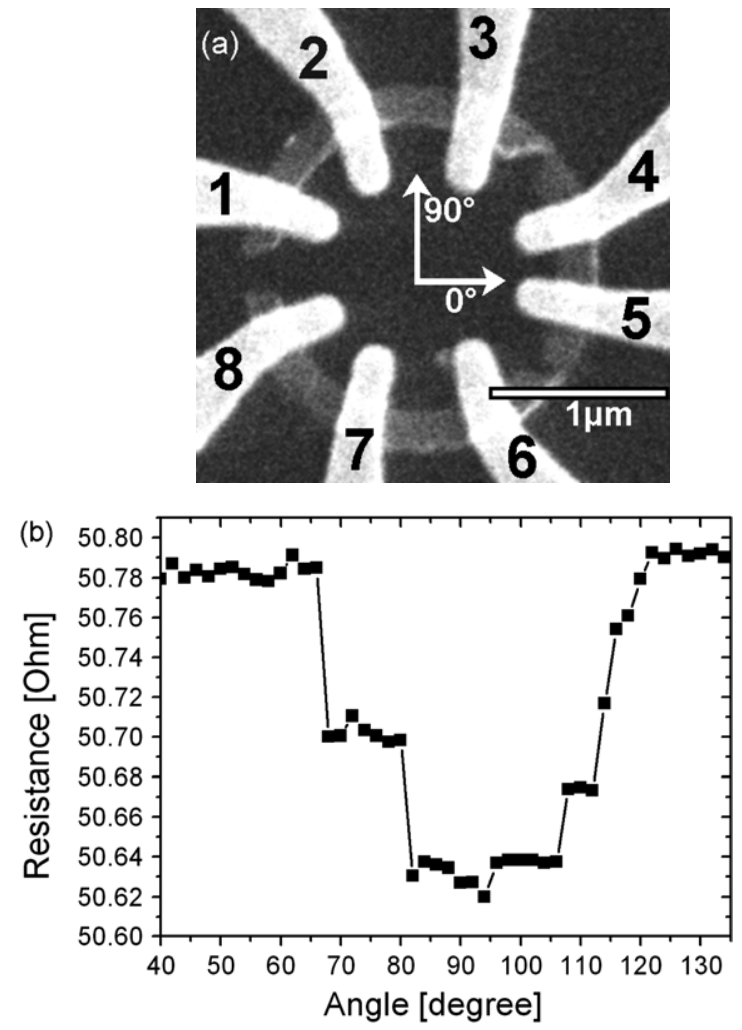

Figure 1. (a) An SEM image of a NiFe ring structure (12 nm thick, $200 \mathrm{~nm}$ wide, $2 \mu \mathrm{m}$ diameter) with the numbered contacts and the polar angles indicated. Constrictions are visible between contacts $3 / 4$ and 5/6 and the ring is severed between contacts 1 and 8 to obtain a well-defined current flow direction. (b) Reference resistance curve taken by saturating the ring and relaxing the field along different field directions to selectively position a domain wall. Low resistance corresponds to the domain wall being located between contacts 2 and 3 and high resistance to the domain wall being located outside the area between the contacts.

Magnetoresistance measurements were carried out in a bath cryostat setup where an external field can be applied in any in-plane direction using vector coils. A standard lock-in technique was used to measure the resistance of a ring section in a four-point configuration. A constant ac current of typically $5 \mu \mathrm{A}$ was applied between contacts 1 and 8 while the voltage drop was measured between contacts 2 and 3 (cf figure $1(a)$ ). The resistance at the voltage contacts depends on whether a domain wall is present in the region between the contacts [3]. At remanence the magnetization inside the domain wall has a component perpendicular to the current flow and therefore reduces the total resistance due to the anisotropic magnetoresistance effect [3]. Reference curves, as shown in figure $1(b)$ for $T_{\text {cryo }}=4 \mathrm{~K}$, were taken by saturating the sample in directions between $40^{\circ}$ and $140^{\circ}$ (cf figure $1(a)$ ) and subsequently relaxing the field to zero and measuring the resistance [3]. These curves allow us to quickly ascertain the wall position by a resistance measurement. The minimum resistance is observed when the domain wall is located fully in between the contacts. Slightly higher levels are obtained when the domain wall is partly located underneath one of the delimiting contacts ( 2 or 3 ) and the maximum resistance is measured when the domain wall is located completely outside the area between contacts 2 and 3 . 

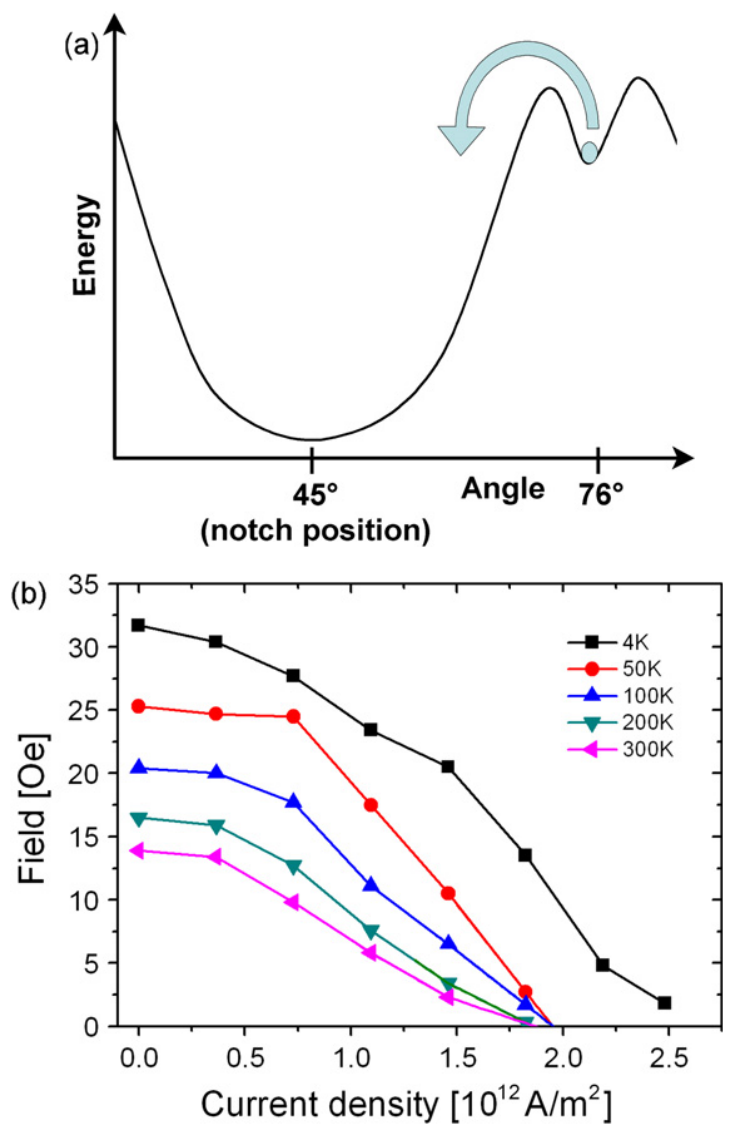

Figure 2. (a) Schematic representation of the potential landscape with the domain wall being located at the edge of the potential well generated by the constriction. The wall is pinned due to natural imperfections, such as the edge roughness. Therefore a potential barrier has to be overcome in order to move the wall into the constriction. (b) Domain wall propagation diagram of the current and field combinations necessary to move the wall into the constriction between contacts 3 and 4 (figure $1(a)$ ) at different temperatures.

In the adjacent area between contacts 3 and 4 , a constriction (width $70 \mathrm{~nm}$ ) is located (figure 1(a)). As shown in [3], transverse walls are dragged towards the constriction by the attractive potential well that the notch generates for the wall. This is because as the wall is moved towards the constriction, its spin structure changes to adapt to the change in the local geometry (as a first approximation the wall simply becomes shorter and therefore lowers its energy as it approaches the constriction). Such a movement can take place without any externally applied fields or currents. Furthermore, the potential well was found to extend far beyond the physical size of the notch [3]. The exact distance from which the domain wall is attracted into the constriction depends not only on the constriction size and shape but also on the domain wall pinning outside the constriction due to defects, such as the edge roughness. In figure $2(a)$ a schematic sketch is shown to visualize the situation.

We have now designed an experiment to probe the combination of current-induced and field-induced domain wall motion with wall motion due to the potential well of the constriction. Current pulses $10 \mu \mathrm{s}$ in length were applied between contacts 1 and 3 , generating current densities of up to $2.5 \times 10^{12} \mathrm{~A} \mathrm{~m}^{-2}$ in the ring structure.
The following experiment was carried out in a sequence of steps typically 10 times for each combination of field strength, current density and temperature. (i) An external field is applied along $76^{\circ}$ and released, so that a domain wall is created at this particular position close to contact 3 in the region between contacts 2 and 3. (ii) The resistance is measured between contacts 2 and 3 . Using the reference curve mentioned before (figure 1(b)), we crosscheck that the domain wall is correctly positioned. (iii) An external magnetic field is applied perpendicularly to the direction of the saturation field along $346^{\circ}$. (iv) A current pulse with electrons flowing from contact 1 to contact 3 is injected. (v) The resistance is measured again. From this resistance measurement we discern whether the domain wall has moved out of the area between the voltage contacts or not.

This procedure was carried out for different combinations of field, current and temperature and we obtain the diagram presented in figure $2(b)$. The different symbols indicate the different cryostat temperatures between 4 and $300 \mathrm{~K}$. For combinations of field and current below the boundary, the wall remains at the original position: for combinations above the boundary the wall is displaced. We define as a critical field and a critical current density the minimum values necessary for moving the wall, so the domain wall is also displaced for values of field and current exactly on the boundary.

At zero current, depending on the temperature, a field of $14-32 \mathrm{Oe}\left(1.1-2.5 \mathrm{kA} \mathrm{m}^{-1}\right)$ is needed to move the domain wall outside the area between the contacts. At current densities of around $2 \times 10^{12} \mathrm{~A} \mathrm{~m}^{-2}$ the wall is moved by the current without any externally applied fields outside the area between the contacts, and in between, a combination of values of field and current displaces the wall as seen in figure $2(b)$.

To check how far the domain wall moves, we have carried out the same experimental sequence (same field and current injected using the same contacts), but this time measuring the resistance between contacts 3 and 4 . We find that at zero current a wall moves into the area between contacts 3 and 4 at $4 \mathrm{~K}$ for a field of $32 \mathrm{Oe}$, which is in line with the observations in figure $2(b)$ and means that the wall moves from its original position between contacts 2 and 3 into the area between contacts 3 and 4 . From the resistance levels we can further deduce (as shown in [3]) that the wall moves directly into the constriction (notch position). The fact that the wall is moved into the constriction is further corroborated by the much larger field ( $>250 \mathrm{Oe} ; 20 \mathrm{kA} \mathrm{m}^{-1}$ ) needed to depin it again from this position.

For the case of current-induced wall motion we find the same behaviour, namely that the wall is displaced into the constriction. This is at first sight surprising, since the current is only injected between contacts 1 and 3 and thus the current can only move the wall underneath contact 3 but not further [18]. From measurements between contacts 3 and 4 , we find however that at $76^{\circ}$ we have positioned the wall at a position just a few degrees outside the attractive potential well of the constriction (as schematically shown in figure $2(a)$ ). This means that it is sufficient to move the wall just a small distance before the movement due to the constriction sets in and pulls the wall into the notch position.

We now turn to the temperature dependence of the propagation fields and currents. The wall experiences pinning 
due to edge irregularities as visible in figure $1(a)$ and consequently a potential landscape such as the one sketched in figure 2(a) results. In order to move the wall into the attractive potential well, this pinning potential has to be overcome. At zero current and a temperature of $4 \mathrm{~K}$ (squares), the field of about $32 \mathrm{Oe}\left(2.5 \mathrm{kA} \mathrm{m}^{-1}\right)$ is needed to move the domain wall into the potential well of the constriction and this field is reduced to about $14 \mathrm{Oe}\left(1.1 \mathrm{kA} \mathrm{m}^{-1}\right)$ at $300 \mathrm{~K}$. For the case of field-induced wall motion, the critical field at zero current thus decreases with increasing temperature (figure $2(b)$ ) as expected, since the increased thermal energy helps to overcome the energy barrier of the pinning potential that separates the wall at $76^{\circ}$ from the potential well of the constriction. The dependence can be described using the following equation from [30]:

$$
H_{\mathrm{d}}(T) \cong H_{0}\left\{1-\left[\frac{k_{\mathrm{B}} T}{E_{0}} \ln \left(\frac{\Gamma_{0} k_{\mathrm{B}} H_{0} T}{1.5 E_{0} v \sqrt{1-H_{\mathrm{d}} / H_{0}}}\right)\right]^{2 / 3}\right\},
$$

where the depinning field measured at $2 \mathrm{~K}$ is taken as $H_{0}$, $v=10 \mathrm{Oe} \mathrm{s}^{-1}$, and the attempt frequency $\Gamma_{0}=10^{10} \mathrm{~Hz}$ [30]. The measured energy barriers of $E_{0} \approx 10^{4} \mathrm{~K}$ correspond well to what was previously observed $[30,38]$, which indicates that thermally assisted depinning of a domain wall is the dominant process.

In the case of high current densities, where the wall is moved by the current without an externally applied field, the temperature dependence of the critical current density for the current-induced domain wall motion is similar to that of the magnetic field in the field-induced case (figure $2(b)$ ). The critical current density decreases with increasing temperature. This means that here thermal activation dominates the temperature dependence similarly to the field-induced case. This is easy to understand taking into account the fact that the wall is located at the edge of the attractive potential well due to the constriction (figure 2(a)). So rather than a large scale propagation, the current only needs to lift the domain wall above the local energy barrier that separates its pinned position (due to edge roughness) from the edge of the potential well (due to the constriction) from whereon the propagation into the constriction is primarily because of the attractive potential well. At higher temperatures thermal activation helps to overcome the potential barrier so that even for smaller current densities the wall moves into the constriction. While this holds qualitatively for all potentials, the quantitative details will depend on the exact shape of the potential. Thus, with this experiment we primarily probe the thermal activation and not the intrinsic temperature dependence of the spin torque effect since no large scale propagation due to the spin torque effect is necessary.

In figure 3(a) an SEM image of a ring with the same width and thickness as in figure 1 but without any artificially defined constrictions is shown. Here no geometrical variations exist (apart from the edge roughness), so that the wall energy does not depend on its position. For an ideal element with no edge roughness, the wall would be moved by an arbitrarily small field and thus there would be no temperature dependence of the critical field (which would always be zero). For the case of current-induced motion this is more complicated and contentious: Tatara and Kohno [24] predict that a critical
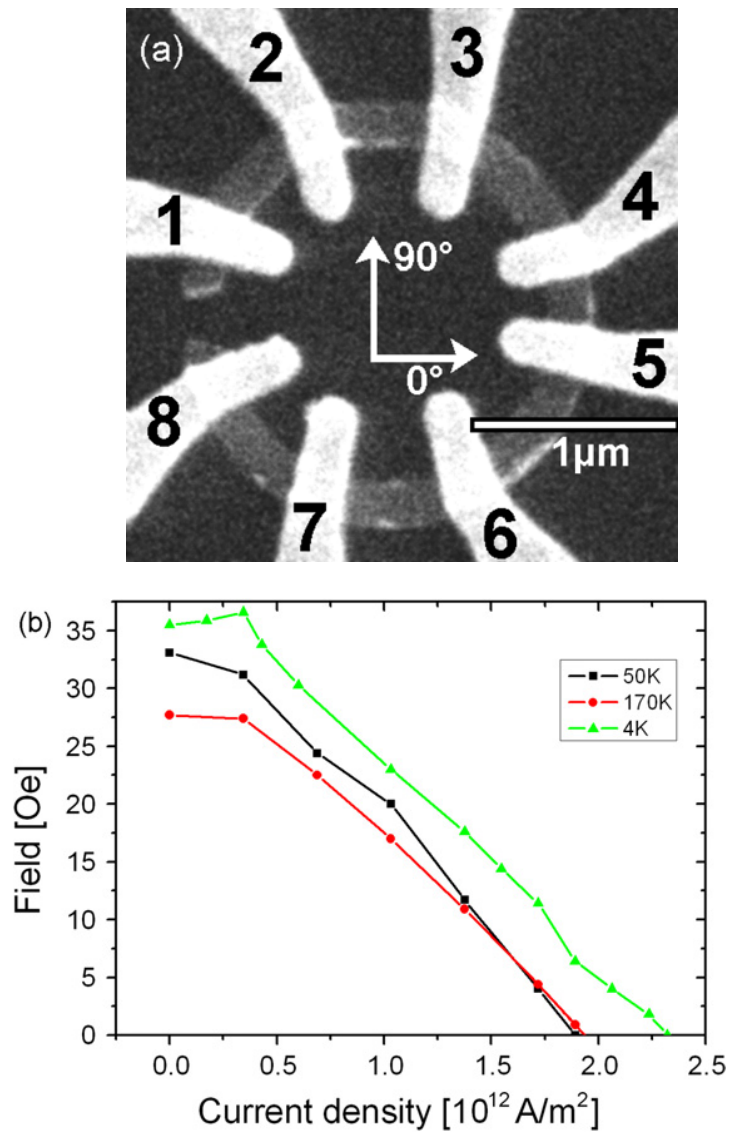

Figure 3. (a) An SEM image of a NiFe ring structure (12 nm thick, $200 \mathrm{~nm}$ wide, $2 \mu \mathrm{m}$ diameter) with no constrictions and with the numbered contacts and the polar angles indicated. $(b)$ Domain wall propagation diagram of the current and field combinations necessary to move the wall at different temperatures in the structure shown in $(a)$.

current density exists even for ideally smooth wires, since the wall is pinned by the transverse anisotropy. Two-dimensional micromagnetic simulations show a critical current density for smooth wires if the non-adiabatic spin torque term is neglected and only the adiabatic spin torque term is taken into account $[26,28]$. If both terms are taken into account, movement is possible even for very small current densities and a critical current density is only predicted for rough wires [28].

Since in this ring only edge roughness and no potential well due to a constriction exist, we can probe not only the influence of temperature due to thermal activation of the domain wall propagation but also the intrinsic temperature dependence of the current-induced wall propagation mechanism. We have measured the resistance between contacts 6 and 7 with the lock-in current and the pulses injected between contacts 8 and 5. The walls were positioned along $258^{\circ}$ as described above. The injected electrons flow clockwise from contact 5 to contact 8 in the ring pushing the wall in the same direction as the external field, which was applied along $168^{\circ}$. In figure $3(b)$ we see the dependence of the critical currents and fields necessary to move a wall in this configuration. At zero current for field-induced wall motion we again find a reduction in the critical field with increasing temperature. For the temperature range considered, we do not expect a variation of the Zeeman-energy $(\propto \boldsymbol{H} \cdot \boldsymbol{M})$, which 
is ultimately responsible for the field-induced domain wall motion since the saturation magnetization is constant far below the Curie temperature (which is $>700 \mathrm{~K}$ ). So for a constant field $\boldsymbol{H}$ the force acting on the wall (and therefore the intrinsic efficiency) will be independent of temperature. Since we observe a decrease in the critical field with temperature we can attribute this to thermal excitations that help overcome the small energy barriers of pinning due to edge roughness.

For the case of large current densities we now see that the boundary lines cross for different temperatures in figure $3(b)$, so that there is no monotonous dependence of the critical current density on temperature. For low temperatures $(4 \mathrm{~K})$ we obtain the largest critical current density. As the temperature is raised the critical current density is reduced as seen for example in the case of $50 \mathrm{~K}$. This reduction can be attributed to thermal activation as described by equation (1). Interestingly, as the temperature is raised further, the critical current density starts to increase again $(170 \mathrm{~K})$ and a non-monotonic behaviour was observed for different positions and fields and current directions of the wall in this geometry. This increase in the critical current density for increasing temperature points to a mechanism that decreases the efficiency of the wall propagation with increasing temperature. Such behaviour was recently observed for a different geometry [38], where it was shown that the critical current density can increase with increasing temperature and this could be due to the intrinsic dependence of the efficiency of the spin torque effect in the current-induced domain wall motion on temperature. This means that for the geometry in figure 3 the dependence of the critical current density on temperature is a superposition of thermal activation (which is dominant at low temperatures and lowers the critical current density when the temperature is increased from 4 to $50 \mathrm{~K}$ ) and the intrinsic temperature dependence of the spin torque effect, which becomes less efficient with increasing temperature and dominates at higher temperatures, leading to an increase in the critical current density as the temperature is increased from 50 to $170 \mathrm{~K}$. It should be noted that the higher critical current density obtained for $170 \mathrm{~K}$ compared with $50 \mathrm{~K}$ is outside the error bars (the relevant error in the field is in general $5-10 \%$ of the current smaller than the symbols used).

In a system where the intrinsic efficiency of the spin torque effect dominates, a monotonous increase in the critical current density with increasing temperature is expected and we now finally review a geometry where such behaviour is observed. In $110 \mathrm{~nm}$ wide and $34 \mathrm{~nm}$ thick rings with vortex walls, we have obtained the data presented in figure 4 [38]. In figure 4(a) we compare the wall propagation field for the field-induced motion at zero current with the case of a high current density, where the wall propagation is primarily due to the current with a density of $2.07 \times 10^{12} \mathrm{~A} \mathrm{~m}^{-2}$. We find for the field-induced propagation a decrease in the propagation field with increasing temperature typical of thermal activation. For high current densities, the opposite behaviour is observed. At $4 \mathrm{~K}$, propagation is obtained with a current density of $2.07 \times 10^{12} \mathrm{~A} \mathrm{~m}^{-2}$ without any externally applied fields, while above $20 \mathrm{~K}$ this current density is not sufficient to move the wall without an externally applied field. With increasing temperature the extra field necessary to move the wall increases, which implies a reduction in the efficiency of
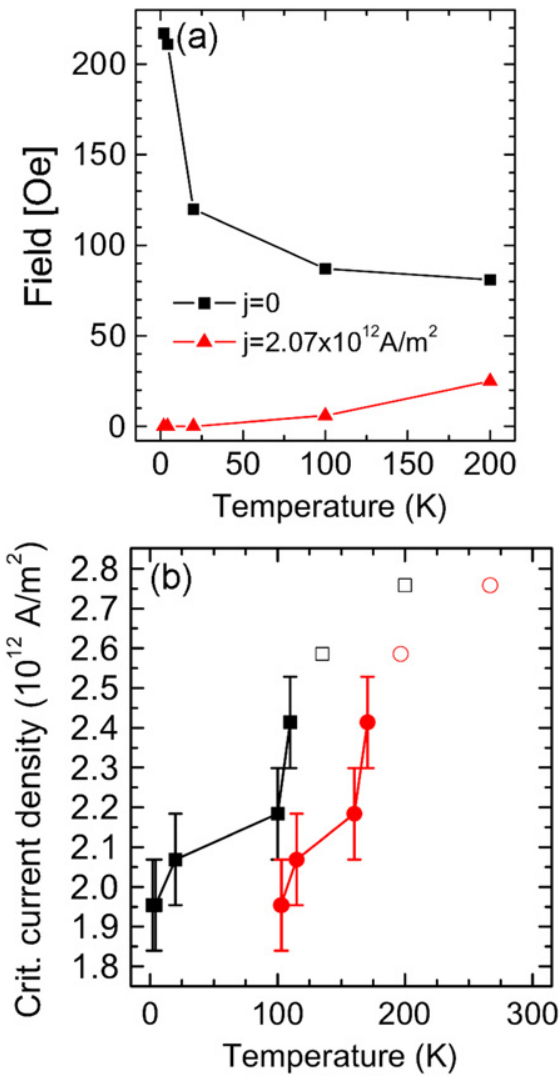

Figure 4. (From [38].) (a) Critical field as a function of the temperature for zero current (black squares) and for $j=2.07 \times 10^{12} \mathrm{~A} \mathrm{~m}^{-2}$ (red triangles). (b) Critical current density as a function of the temperature. Black squares refer to the cryostat temperature $T_{\text {cryo }}$, red circles to real temperatures $T_{\text {real }}$ corrected for the effect of Joule heating. Open symbols indicate maximum values of the current density injected during the sample lifetime and are therefore lower limits for the critical current density.

the spin torque effect. This reduction in the efficiency becomes even more obvious when we look at the critical current density for pure current-induced domain wall motion as a function of temperature. As plotted in figure 4(b), the critical current density monotonously increases with increasing temperature. We have further measured the temperature rise during the pulse injection and in figure $4(b)$ the critical current density is also plotted for the real sample temperature (discs) rather than the cryostat temperature (squares) ${ }^{5}$. Since from the real sample temperature we can directly derive the thermal excitations $\left(\approx k_{\mathrm{B}} T\right)$ and since the critical current density increases with increasing real sample temperature, we can conclude that for this system thermally activated wall motion is not dominating and rather a physical mechanism must exist that renders the spin torque in the current-induced domain wall motion intrinsically less efficient at higher temperatures.

To our knowledge, only Tatara et al have so far included thermal effects in the theoretical description of current-driven domain wall motion [31], and they do not predict an increase in the critical current density with temperature. The strength of the spin torque effect was predicted to depend on the nonadiabaticity parameter [29]. The non-adiabaticity parameter

5 The Joule heating due to the current pulses was determined to be smaller than $150 \mathrm{~K}$ for all temperatures for the maximum current density so that the sample temperature is always well below the Curie temperature. 
depends on the square of the exchange length divided by the square of the spin flip length and we would expect that for higher temperatures the spin flip length is reduced and the exchange length should stay constant in the temperature regime that we probe. Thus, at higher temperatures a larger non-adiabaticity parameter is expected, which is predicted to make the spin torque effect more efficient, in contrast to our observation. Since we observe an increase in the critical current density with temperature, we conclude that another mechanism must exist, which reduces the efficiency of the spin torque exerted on the domain wall at increased temperature. Spinwave generation can be such a mechanism if a symmetry breaking between magnon excitation and annihilation for different directions occurs due to the current flow.

When taking into account spin currents, it was theoretically predicted $[32,33]$ that the spin wave dispersion $\omega(\boldsymbol{k})$ becomes asymmetric and can be expressed as $\omega(\boldsymbol{k})=$ $\omega_{0}+f_{0} \boldsymbol{k}^{2}+\boldsymbol{f}_{\mathbf{1}}\left(I_{\mathrm{s}}\right) \cdot \boldsymbol{k}$ with the spin current $I_{\mathrm{s}}$, a constant $f_{0}$ and a function $f_{1}\left(I_{\mathrm{s}}\right)$ of the spin current. Also due to this asymmetry, the magnon density of states becomes asymmetric with respect to $\boldsymbol{k}$ so that for non-zero temperature the number of thermally excited spinwaves becomes asymmetric as well in the presence of a current. This effect has interesting implications at very low temperatures: since the spin wave gap at room temperature is of the order of a few tens of meV corresponding to a few $\mathrm{GHz}$ [39] this means that at milli-Kelvin temperatures the population of spin waves is extremely asymmetric: only spin waves with $\boldsymbol{k}$ vectors in one direction are excited. At room temperature though this effect is much smaller, since it is smeared out due to thermal excitations, which are much larger than the spin wave gap. Though spin waves can play a crucial role even at higher temperatures, since they might reduce the polarization of the current: in a simple single parabolic band model with the two spin direction bands energy-split and a spherical Fermisurface, the levels of occupation for positive and negative Fermi wave vectors $\boldsymbol{k}$ are different due to the current flowing (shift of a spherical Fermi-surface). If thermally excited magnons are present, then the current-carrying electrons can primarily absorb magnons with a wave vector $\boldsymbol{q}$ that flips an electron from a majority to a minority one, so that effective polarization of the current is reduced.

In conclusion, we have investigated field- and currentinduced domain wall propagation in permalloy nanostructures at variable temperatures. Introducing constrictions, which generate attractive potential wells for transverse walls, we find that domain walls can be moved from a position outside the potential well into the constriction using fields and currents. If currents are injected into a contact, which lies between the domain wall position and the constriction, the current can move the domain wall to the edge of the potential well, from where the movement into the constriction occurs due to the attractive potential well. The temperature dependence for both the field- and the current-induced case shows that thermal activation is dominating with critical currents and fields being reduced for higher temperatures. For ring structures with no constrictions, field-induced motion is again aided by thermal excitations. Critical currents for current-induced motion exhibit a non-monotonous dependence on temperature with the critical current density being reduced when the temperature is raised from 4 to $50 \mathrm{~K}$ due to thermal excitations, while it increases as the temperature is raised further to $170 \mathrm{~K}$. This increase has to be attributed to the intrinsic dependence of the efficiency of the spin torque effect for current-induced domain wall motion on temperature. This dependence is even more obvious for a geometry with vortex walls, where the critical current density increases with temperature for all temperatures. A possible reason for this dependence is the role of thermally excited spin waves, which due to an asymmetry in the spin wave spectrum can render the spin torque effect less efficient at higher temperatures where more spin waves are excited.

\section{Acknowledgments}

The authors acknowledge the support given by the Deutsche Forschungsgemeinschaft through SFB 513, the Landesstiftung Baden-Württemberg, the EPSRC (UK), the 'Région Ile de France', and the 'Conseil Général de l'Essonne'. Technical support by E Cambril is gratefully acknowledged.

\section{References}

[1] McMichael R D and Donahue M J 1997 IEEE Trans. Magn. 334167

[2] Laufenberg M et al 2006 Appl. Phys. Lett. 8852507

[3] Kläui M et al 2003 Phys. Rev. Lett. 9097202

[4] Faulkner C C et al 2004 J. Appl. Phys. 956716

[5] Kläui M et al 2005 Appl. Phys. Lett. 87102509

[6] Himeno A et al 2006 J. Appl. Phys. 9908 G304

[7] Beach G S D et al 2005 Nature Mater. 4741

[8] Nakatani Y et al 2003 Nature Mater. 2521

[9] Cowburn R P 2004 Patents PCT/GB2003/001266, PCT/GB2004/000840

[10] Allwood D A et al 2005 Science 3091688

[11] Berger L 1984 J. Appl. Phys. 551954

[12] Slonczewski J C 1996 J. Magn. Magn. Mater. 159 L1

[13] Freitas P P and Berger L 1985 J. Appl. Phys. 571266

[14] Yamaguchi A et al 2004 Phys. Rev. Lett. 9277205

[15] Kläui M et al 2005 Phys. Rev. Lett. 9526601 Kläui M et al 2006 Appl. Phys. Lett. 88232507

[16] Grollier J et al 2003 Appl. Phys. Lett. 83509

[17] Vernier N et al 2004 Europhys. Lett. 65526

[18] Kläui M et al 2005 Phys. Rev. Lett. 94106601

[19] Ravelosona D et al 2005 Phys. Rev. Lett. 95117203

[20] Parkin S S P 2003 US Patent 6,834,005

[21] Yamaguchi A et al 2005 Appl. Phys. Lett. 8612511

[22] Himeno A, Kasai S and Ono T 2005 Appl. Phys. Lett. 87243108

[23] Gopar V A et al 2004 Phys. Rev. B 6914426

[24] Tatara G and Kohno H 2004 Phys. Rev. Lett. 9286601

[25] Li Z and Zhang S 2004 Phys. Rev. Lett. 92207203

[26] Thiaville A, Nakatani Y, Miltat J and Vernier N 2004 J. Appl. Phys. 957049

[27] Zhang S and Li Z 2004 Phys. Rev. Lett. 93127204

[28] Thiaville A, Nakatani Y, Miltat J and Suzuki Y 2005 Europhys. Lett. 69990

[29] He J, Li Z and Zhang S 2005 J. Appl. Phys. 9816108

[30] Himeno A et al 2005 J. Magn. Magn. Mater. 286167

[31] Tatara G, Vernier N and Ferré J 2005 Appl. Phys. Lett. 86252509

[32] Bazaliy Y B, Jones B A and Zhang S-C 1998 Phys. Rev. B 57 R3213

[33] Fernández-Rossier J et al 2004 Phys. Rev. B 69174412

[34] Kläui M et al 2002 Appl. Phys. Lett. 81108

[35] Kläui M et al 2003 J. Phys. Condens. Matter 15 R985

[36] Kläui M et al 2004 Appl. Phys. Lett. 855637

[37] Kläui M et al 2004 Physica B $\mathbf{3 4 3} 343$

[38] Laufenberg M et al 2006 Phys. Rev. Lett. 9746602

[39] Gubbiotti G et al 2003 IEEE Trans. Magn. 392750 